\title{
Developing Collaborative Applications On the World Wide Web
}

\section{Andreas Girgensohn}

\author{
FX Palo Alto Laboratory \\ 3400 Hillview Avenue \\ Palo Alto, CA 94304, USA \\ +16508137244 \\ andreasg@pal.xerox.com
}

\author{
Alison Lee \\ IBM T.J. Watson Research Center \\ P.O. Box 704 \\ Yorktown Heights, NY 10598 \\ +19147847712 \\ alisonl@us.ibm.com
}

\begin{abstract}
The World Wide Web is often viewed as the latest and most user friendly way of providing information over the Internet (i.e., server of documents). It is not customarily viewed as a platform for developing and deploying applications. In this tutorial, we introduce, demonstrate, and discuss how Web technologies like CGI scripts, Javascript, and Java can be used in combination with Web browsers to design, create, distribute and execute collaborative applications. We discuss constraints with the Web approach as well as recent extensions that support application development.
\end{abstract}

\section{Keywords}

Collaborative applications, interactive applications, forms, HTML, MIME, CGI, HTTP, URL, Java, JavaScript, web server, web browsers, cookies, proxy servers, software development, chat, desktop video conference, voting application, group calendar, multi-user games.

\section{INTRODUCTION}

In the last few years, our community has seen phenomenal interest and growth of the World Wide Web as a userfriendly means for accessing and disseminating information. Whether it is for internal or external use, the Web enables individuals, researchers, and companies to communicate in an easy-to-use and cost-effective manner compared to "commercial" but proprietary or research-prototype systems. However, what is less known about the Web is its appropriateness as a development platform for highly interactive information systems and groupware applications [3, 5]. Drawing on our experiences at NYNEX and FX Palo Alto Laboratory and using concrete examples, we demonstrate how to develop collaborative applications using the components of the Web (e.g., CGI scripts, JavaScript, Java).

The goal of the tutorial is to provide insights into how to use the Web as a rapid prototyping and development platform for collaborative applications. At the end of the tutorial, participants will have a basic understanding to begin developing collaborative applications using this platform.

\section{THE WEB AS A DEVELOPMENT PLATFORM}

There are several appealing aspects to using the Web for developing collaborative applications. First, standard browsers such as Netscape Navigator and Microsoft Internet Explorer are available for many hardware platforms (PC, Mac, Sun, SGI). This enables users to run collaborative applications on any platform from any network location. Second, the Web provides many components and features that a developer can use to implement user interface functionality, to access and transport information over networks, to link to commercial databases, specialized search engines, etc., and to view foreign document formats. Such features would otherwise need to be implemented from scratch. Third, Web developers and users do not need to be concerned about software installation or different versions of the application in use. All in all, the Web reduces the turnaround time for software development with its support for development, testing and debugging, maintenance, and distribution of the resulting collaborative application.

Unlike specialized or proprietary groupware toolkits or groupware development environments, applications developed with the Web and accessed from the Web are easier-touse, easier-to-install, easier-to-access, easier-to-develop, and easier-to-maintain. Consequently, developers of collaborative applications can focus on the task of working with users to identify, implement, and iterate over the capabilities of the collaborative applications. Users have the opportunity, early in the development effort, to interact with and provide feedback on the usability, utility and acceptability of the application.

\section{CONTENT}

The tutorial introduces the particular kinds of collaborative applications that are the focus of the tutorial. We also motivate why the Web is an appropriate development environment for collaborative applications.

After a short introduction, the tutorial is broken up into a morning and afternoon session consisting of formal presentations and small group discussions. The formal presentation introduces Web tools and techniques and demonstrates how they can be use to build a number of examples of small collaborative applications. For instance, we illustrate how a proxy server can add awareness information that allows users to see who else is looking at the same document. The topics covered in the presentation are:

- the set of standards (HTML, URL, HTTP, MIME, CGI) that enable the development and delivery of applications,

- the Web technologies (i.e., HTML, forms, tables, frames, imagemaps, dynamic HTML) that can be used easily to create user interfaces and support user interactions, 
- the building blocks for programming the server-side of an application (e.g., CGI scripts, cookies, proxy server), as well as the engineering considerations (e.g., application delivery, maintenance, cross-platform compatibility, user authentication, proxy servers),

- the building blocks available for programming the clientside of an application (e.g., Java, Javascript), as well as a discussion on supporting interaction between different building blocks (e.g., combining Java and JavaScript) and mechanisms to integrate non-Web components into the Web framework (e.g., plug-ins, helper applications).

As each topic is introduced and using one of a number of examples of small collaborative applications, we illustrate how the Web building blocks can be used for application development. The examples include tools to:

- display a calendar view containing user and group activities using tables, GIF library, and CGI script,

- enter and modify several daily activities using HTML forms and JavaScript,

- enter, modify, annotate and comment on meeting notes using Java-based Dynamic Forms [2] and CGI scripts,

- facilitate desktop video conferencing using helper applications and CGI scripts,

- engage in synchronous chat using CGI scripts, Java applets, Javascript, and Remote Method Invocation,

- vote on issues using CGI Forms, cookies, and Java applets,

- play a turn-taking word game involving periodic polling, automatic form submission, and page reload, and

- be made aware of other people viewing the same document using a proxy server.

In the small group discussions (one in the morning and one in the afternoon), tutorial attendees will break off into small groups to work on the two exercises. The first exercise is to identify and design an interactive and collaborative application. The second exercise is to identify how they would develop all or part of the application from the first exercise.

The tutorial concludes with a discussion of the limitations of the Web approach and a presentation of the enhancements to the Web.

\section{OBJECTIVES}

At the end of the tutorial, participants will gain a basic understanding of:
- the software concepts and architecture of the Web

- the tools and technologies underlying the Web

- the issues in designing and developing applications

- how to use the Web to begin developing collaborative applications using this platform.

\section{AUDIENCE}

This tutorial is targeted at an intermediate to advanced level audience with a focus on how to actually build collaborative applications. It is intended for researchers, designers, and developers working on CSCW applications or creating Web content with an interactive and collaborative component. Attendees should be familiar with the World Wide Web and have some experience in using a Web browser. Basic familiarity with at least one programming language with a $\mathrm{C}$-like syntax $(\mathrm{C}++$, Java, Perl) is assumed. No development experience in $\mathrm{CSCW}$ is required. However, attendees should be comfortable with seeing code fragments.

\section{REFERENCES}

1. M.E. Atwood, B. Burns, D. Gairing, A. Girgensohn, A. Lee, T. Turner, S. Alteras-Webb, and B. Zimmermann. Facilitating Communication in Software Development. In Symposium on Designing Interactive Systems. 1995. New York: ACM. pp. 65-73.

2. A. Girgensohn and A. Lee. Seamless Integration of Interactive Forms into the Web. In Proceedings for the Sixth International World Wide Web Conference, 1997, pp. 619-629.

3. A. Girgensohn, A. Lee, and K. Schlueter. Experiences in Developing Collaborative Applications Using the World Wide Web "Shell”. In Hypertext'96 Conference Proceedings. 1996. New York: ACM. pp. 246-255.

4. A. Lee, A. Girgensohn, and K. Schlueter. NYNEX Portholes: Initial User Reactions and Redesign Implications. In GROUP'97, Proceedings of the International ACM SIGGROUP Conference on Supporting Group Work. 1997. New York: ACM. pp. 385-394.

5. J. Rice, A. Farquhar, P. Piernot, and T. Gruber. Using the Web Instead of a Window System. In CHI'96 Conference Proceedings (Vancouver, Canada). 1996. New York: ACM. pp. 103-110. 